\title{
Manipulation of the Walrasian mechanism in production economies with unbounded short-selling
}

\author{
Laurence Kranich \\ Department of Economics, University at Albany, SUNY, 1400 Washington, Ave., Albany, \\ NY 12222-1000, USA (e-mail: L.Kranich@albany.edu)
}

Received: 17 January 2003 / Accepted: 4 April 2005

Rev. Econ. Design (2005) DOI 10.1007/s10058-005-0123-0

Unfortunately the publisher failed to print the article correctly. The corrected version is given here

\begin{abstract}
Hurwicz (1979) and Otani and Sicilian (1982, 1990) characterized the Nash equilibrium allocations of the Walrasian demand manipulation game in successively more general exchange environments. In this paper, I extend the analysis to production economies with short-selling. First, I generalize Hurwicz's and Otani and Sicilian's theorem that any allocation at which each agent's consumption bundle lies above her true offer curve can be supported in Nash equilibrium. I then show that for finite economies of any size the set of such allocations is often topologically large.
\end{abstract}

JEL classification: D51, D82

Key words: Manipulation, Walrasian mechanism, production, short-selling

\section{Introduction}

In this paper, I study the Walrasian demand manipulation game in production economies with unbounded short-selling. ${ }^{1}$ This extends previous work by Hurwicz

For comments on this and earlier versions of the paper, I wish to thank Rick Bond, Bhaskar Chakravorti, Tom Gresik, Costas Syropoulos and William Thomson. I would especially like to thank Mike Jerison for helping to overcome a difficulty with a previous version. Also, the comments of the anonymous referees are gratefully acknowledged.

1 Here, I use the expressions demand manipulation and preference manipulation interchangeably. Since the present paper focuses exclusively on the Walrasian mechanism, it is sufficient to consider the relevant manifestion of preferences, namely, demand. Also, in keeping with the antecedent literature, I refer to this as a game although, properly speaking, it is a game form since it specifies an outcome function (in this case, correspondence) rather than a payoff function. 
(1979) and Otani and Sicilian (1982, 1990) who considered successively more general exchange environments. I focus on convex, private ownership economies with a single (aggregate) technology, but the techniques are easily generalized to include additional firms. First, I establish that any allocation at which each agent's consumption bundle lies above its true offer curve can be supported as a Nash equilibrium allocation. ${ }^{2,3}$ Here, however, the notion of an offer curve is modified to incorporate the effect of prices on wealth. I then show that for finite economies of any size which satisfy additional smoothness and regularity conditions the set of such allocations is topologically large in that it contains an open subset of the feasible set. Thus, even in large (finite) economies, strategic behavior may remain a significant problem. These results are immediately applicable to financial and commodities markets, where short-selling is commonplace ${ }^{4}$, and, interpreted appropriately, they may pertain to other cases as well (see Footnote 10, below).

In his classic paper, Samuelson (1954) first observed that under the competitive mechanism agents would have the incentive to misrepresent their demands for public goods. But Hurwicz (1972) showed that such incentives are not limited to economies with public goods; they arise in finite, private good economies as well.

The fact that strategic behavior may cause distortions in the competitive mechanism has led some authors to evaluate the limiting incentive compatibility of the mechanism, that is, to evaluate the incentive to deviate from true competitive behavior as the economy becomes large. ${ }^{5}$ First, Roberts and Postlewaite (1976) showed that for sufficiently well-behaved economies the potential utility gain from unilater-

2 To be precise, I consider a generalization of the notion of Nash equilibrium to games with outcome correspondences (see Sect. 3, below).

3 A word concerning the information structure and the equilibrium notion:

In his seminal article, Hurwicz (1972) considered not only the Walrasian mechanism but all informationally decentralized mechanisms, that is, those in which each agent knows only its own characteristics. Specifically, he investigated the conflict between informational decentralization and incentives (together with individual rationality and Pareto efficiency), where the latter was formalized by requiring that truthful revelation be a Nash equilibrium for every profile of characteristics. Since then numerous authors have adopted the same information/equilibrium structure (including Otani and Sicilian 1982, 1990). (See the discussion in Postlewaite 1985.) The appropriateness of doing so has been the subject of extensive discussion, and there have been various attempts to rationalize this combination (cf., Hurwicz 1972; Maskin 1985; Thomson 1984; particularly Postlewaite 1985; Groves and Ledyard 1987). In keeping with the antecedent literature, I adopt Hurwicz's informational setting. But that is not necessary; one could view the model as involving an uninformed planner with otherwise well informed agents (see Sobel 1981, 1998, for example).

4 Indeed, there have been several well publicized attempts to manipulate market outcomes, including the Hunt brothers' attempt to corner the silver market in 1979 and the attempt by Salomon Brothers Inc., among others, to manipulate U.S. Treasury bond auctions in 1991 (see Wall Street Journal, November 14, 1998, p. C28 and March 31, 1994, p. C1, respectively).

5 Another question concerns the implementability of the true Walrasian outcomes by means of an alternative game form (see Postlewaite 1985, for a general discussion of Nash implementation in exchange environments, and see Hong 1995, for an extension to production economies). While this is clearly an important question, it does not diminish the need to study the Walrasian manipulation game. Rather, implementation addresses the normative concern of whether it is possible to achieve the true Walrasian outcomes. Whereas, manipulation concerns a positive issue: given that the market mechanism is used to allocate resources, what are the likely outcomes when agents behave strategically, or when they attempt to distort prices through their demand or supply behavior? (This is particularly relevant in light of such actual attempts as those described in Footnote 4.) 
ally misrepresenting preferences diminishes as the economy grows. Subsequently, Jackson (1992) established that if other agents announce their true demands, then the optimal demand announcement actually converges to the true competitive demand.

While these results establish that truthful behavior approximately constitutes a Nash equilibrium in large economies, they do not rule out the possibility that there are other Nash equilibria some of which may be quite different from the true competitive demands. ${ }^{6}$ Hurwicz (1979) and Otani and Sicilian $(1982,1990)$ showed that that is indeed the case in finite exchange environments.

First, Hurwicz (1979) characterized the Nash equilibrium allocations of the preference manipulation game in 2-agent, 2-commodity exchange economies as those allocations at which each agent's consumption bundle lies above its true offer curve. ${ }^{7}$ Next, Otani and Sicilian (1982) generalized this characterization to allow additional agents or additional commodities, but not both. And finally, for the general n-agent, m-commodity case, Otani and Sicilian (1990) (henceforth OS) established that all such allocations can be supported in Nash equilibrium and, moreover, that the set of such allocations is topologically large. ${ }^{8}$

In this paper, I extend the analysis to production economies with short-selling. ${ }^{9}$ While short-selling is common in financial and commodity markets, it is allowed here more generally. ${ }^{10,11}$ First, I modify the notion of an offer curve to incorporate the effect of prices on wealth. I then generalize the constructive argument in OS to show that any allocation at which each agent's consumption bundle lies above its

6 See also Jackson (1992) on this point.

7 Thomson (1979) established an analogous result for the Lindahl preference manipulation game in economies with public goods (see also Otani 2001). For a systematic study of the manipulability of more general economic mechanisms see Thomson (1984).

8 More recently, Otani (1996) considered the issue of indeterminacy of strategic equilibria in the general $n \times m$ model.

9 In light of Rader's (1972) Principle of Equivalence (p. 260) between production economies and exchange economies once the former are interpreted in terms of net trades, it might seem that such an extension is to be expected. However, the present extension relaxes the nonnegativity constraint on consumption, thus allowing short-selling (indeed, short-selling is necessary in the present context - see Footnote 25, below). Since this admits greater opportunities to deviate, it suggests that the set of Nash equilibria might shrink relative to the exchange counterpart without short-selling. Nevertheless, it is shown below that the structure of the set of Nash equilibrium outcomes is the same as in the exchange context.

Also, Rader's equivalence result does not incorporate strategic considerations in associating an appropriate exchange environment with each general economy and hence does not pertain, as stated.

Finally, I would point out that the concept of a modified endowment and the associated offer curve introduced below are purely artifactual, based as they are on fictitious private production sets which need not represent actual production possibilities available to the agents. Therefore, in generalizing the Hurwicz-Otani-Sicilian result, the challenge lies in identifying the appropriate analogues to bound the set of Nash equilibrium outcomes.

10 One might interpret the model as describing the spot market-clearing process in an intertemporal setting. There, short-selling entails the promise to deliver units at a later date and thus allows us to relax the instantaneous resource constraints.

11 Papers that have incorporated unbounded short-selling in full general equilibrium models include Werner (1987); Nielsen (1989); Cheng (1991); Chichilnisky and Heal (1993); Dana et al. (1997); Chichilnisky (1993); Page and Wooders (1994); and Brown and Werner (1995). 
true modified offer curve can be supported as a Nash equilibrium allocation. I also establish that for all finite economies which are smooth and for which the technology exhibits sufficient curvature, the set of such allocations remains topologically large.

The paper thus partially characterizes those outcomes which are likely to emerge under manipulative/strategic behavior and argues that such behavior may remain a significant problem even in large (finite) economies. Some applications of our study include manipulation in financial/asset markets, strategic trade policy, and speculation in markets for foreign exchange.

The remainder of the paper is organized as follows. The next section contains notation and definitions as well as a description of the class of economies under consideration. In Sect. 3, I define the strategic aspects of the model. Section 4 contains our main results. Finally, Sect. 5 contains a brief conclusion.

\section{Preliminaries}

Let $N=\{1, \ldots, n\}$ denote the set of consumers and $M=\{1, \ldots, m\}$ the set of commodities. I assume $n$ and $m$ are finite. Consumers are indexed by $i$ and $j$, and commodities are indexed by $k$. The production sector is described by the aggregate production set $Y \subset \mathbb{R}^{m} \cdot{ }^{12}$ Consumer $i$ owns a share $\theta^{i}$ of the production sector. ( $\theta^{i} \geq 0$ for all $i$, and $\sum_{i \in N} \theta^{i}=1$ ). In addition to its ownership share, $i$ is characterized by its consumption set $X^{i} \subseteq \mathbb{R}^{m}$, its initial endowment $\omega^{i} \in X^{i}$, and its (true) preference relation $\widehat{\succsim}_{i} \subset X^{i} \times X^{i}$. For simplicity, and since I wish to allow unlimited short-selling, I take $X^{i}$ to be the entire set $\mathbb{R}^{m}$.

The economy is described by the list $e=\left\{\left(X^{i}, \omega^{i}, \theta^{i}, \widehat{\succsim}_{i}\right)_{i \in N}, Y\right\}$. With the exception of $\left(\widehat{\succsim}_{i}\right)_{i \in N}, e$ is assumed to be common knowledge.

I impose the following restrictions on $e$ :

Assumption 1. For all $i \in N, \widehat{\succsim}_{i}$ is complete, reflexive, transitive, continuous, strictly convex and strictly monotonic.

Assumption 2. $Y$ is closed, strictly convex and satisfies $-\mathbb{R}_{+}^{m} \subset Y$ and $Y \cap \mathbb{R}_{+}^{m}=$ $\{0\}{ }^{13}$

These assumptions are standard. ${ }^{14}$

Let $\omega=\left(\omega^{1}, \ldots, \omega^{n}\right), \Omega=\sum_{i \in N} \omega^{i}$ and $X=X^{1} \times \ldots \times X^{n}$ with generic element $x=\left(x^{1}, \ldots, x^{n}\right)$. Also, let $X^{-i}=X^{1} \times \ldots \times X^{i-1} \times X^{i+1} \times \ldots \times X^{n}$.

\footnotetext{
12 I adopt the following notational conventions. $\mathbb{R}^{m}$ denotes the $m$-dimensional Euclidean space, and $\mathbb{R}_{+}^{m}$ and $\mathbb{R}_{++}^{m}$ denote, respectively, its nonnegative and positive orthants. The vector inequality $x \geq y$ means $x_{k} \geq y_{k}$ for all $k ; x>y$ means $x_{k} \geq y_{k}$ for all $k$ and $x \neq y$; and $x \gg y$ means $x_{k}>y_{k}$ for all $k$. $\partial A$ denotes the topological boundary of the set $A$, int $A$ denotes the interior of $A$, and $\operatorname{dim} A$ denotes its dimension. Finally, $v^{t}$ denotes the transpose of the column vector $v$.

13 In Kranich (1992) I formally established that the Walrasian mechanism is incentive compatible in production economies with linear technologies. See also Zhou (1991).

14 Since consumption is unbounded below, the assumptions are not sufficient to ensure the existence of Walrasian equilibrium. However, the main argument in the paper does not require that an equilibrium exists for either the true economy or for every purported one. This is discussed in detail in Footnote 23 and the adjoining text. Note that in the constructive proof of the main result an equilibrium does exist for the purported economy appearing there.
} 
Other expressions involving " $-i$ " are defined analogously. A feasible allocation for $e$ is a list $(x, y) \in X \times Y$ such that $\sum_{i \in N} x^{i} \leq y+\Omega$. Let $F$ denote the feasible set, and let $\bar{F}$ denote the restriction of $F$ to $X \times \partial Y$. Since $\Omega$ and $Y$ are common knowledge, I take $F$ to be known as well.

To facilitate the analysis, I take commodity $m$ to be numeraire and normalize its price to 1 . Also, I restrict attention to positive prices. Thus, a price vector is a list $p=\left(p_{1}, \ldots, p_{m-1}, 1\right)$ such that $p_{k}>0$ for all $k=1, \ldots, m-1$. Let $P$ denote the set of price vectors.

Generally, supply is determined by maximizing profit, or by solving

$$
\text { maximize } p \cdot y \text { subject to } y \in Y \text {. }
$$

However, for an arbitrary $p \in P$, there may be no solution to (1). Therefore, let $\bar{P} \subseteq P$ denote the set of prices for which such a maximum does exist. The supply correspondence, $\eta$, associates with each $p \in \bar{P}$ the set of production plans that solve (1). The maximum profits are denoted by $\pi(p)$. Under Assumption $2, \pi(p)$ is well-defined and nonnegative for all $p \in \bar{P}$ and $\eta(p)$ is single-valued.

Next, let $\widehat{d^{i}}(p, I)$ denote agent $i$ 's true demand at prices $p$ and income $I$. That is, $\widehat{d^{i}}(p, I)$ is the set of $\widehat{\succsim}_{i}$-maximal elements of $B^{i}(p, I) \equiv\left\{x^{i} \in X^{i} \mid p \cdot x^{i} \leq I\right\}$. Since $X^{i}$ is unbounded below, here, too, such a maximal element need not exist for an arbitrary $p \in P$. Hence, the domain of $\widehat{d}^{i}$ is restricted to the set $P^{i} \subseteq P$ for which a maximum does exist. Under Assumption $1, \widehat{d}^{i}: P^{i} \times \mathbb{R} \rightarrow X^{i}$ is well-defined and single-valued.

Here, wealth is determined endogenously such that at prices $p \in \bar{P}, I^{i}(p) \equiv$ $p \cdot \omega^{i}+\theta^{i} \pi(p)$. Although a slight abuse of notation, I denote the respective reducedform budget and demand expressions by $B^{i}(p) \equiv B^{i}\left(p, I^{i}(p)\right)$, for $p \in \bar{P}$, and by $\widehat{d^{i}}(p) \equiv \widehat{d^{i}}\left(p, I^{i}(p)\right)$, for $p \in \bar{P}^{i} \equiv P^{i} \cap \bar{P}$.

Next, define agent $i$ 's private production possibility set by $Y^{i} \equiv\left\{\omega^{i}\right\}+\theta^{i} Y$. $Y^{i}$ is the set of consumption vectors that would be available to agent $i$ if it had access to the technology $\theta^{i} Y^{15}$ (see Fig. 1 for the case in which $n=m=2$ and $\left.\Omega_{2}=0\right)$. At prices $p \in \bar{P}$, $i$ 's modified endowment is $o^{i}(p) \equiv \omega^{i}+\theta^{i} \eta(p)$. Notice that $o^{i}(p)$ lies on the boundary of $Y^{i}$ and is supported by $p$. Agent $i$ 's wealth is given by $p \cdot o^{i}(p)=p \cdot \omega^{i}+\theta^{i} p \cdot \eta(p)$. Thus, $B^{i}(p)$ is determined by the supporting hyperplane to $Y^{i}$ at $o^{i}(p)$.

For later reference, I also depict the reduced-form demand function $\widehat{d}^{i}$ in Fig. $1 .{ }^{16}$ First, since preferences are monotonic, $p \cdot \widehat{d^{i}}(p)=p \cdot o^{i}(p)$, for all $p \in \bar{P}^{i}$. Let $\bar{x}^{i}$ denote the unique $\widehat{\succsim}_{i}$-maximal element of $Y^{i}$ (providing it exists), which I refer to as $i$ 's autarkic consumption, and let $\bar{p}^{i}$ denote the supporting prices at $\bar{x}^{i}$.

15 See Gabszewicz and Vial (1972).

16 In the exchange context, $i$ 's offer curve would emanate from its initial endowment $\omega^{i}$. Indeed, there, one can think of $\widehat{d^{i}}(p)$ as $i$ 's demand from $\omega^{i}$. Here, too, one can think of $\widehat{d^{i}}(p)$ as $i$ 's demand from the (modified) endowment $o^{i}(p)$, but the latter varies with $p$ as well. This renders the depiction of $\widehat{d^{i}}$ in $\mathbb{R}^{2}$ somewhat more complicated. In particular, as described below, the intersection between a budget line and an offer curve does not necessarily correspond to a preference maximizing consumption bundle. 


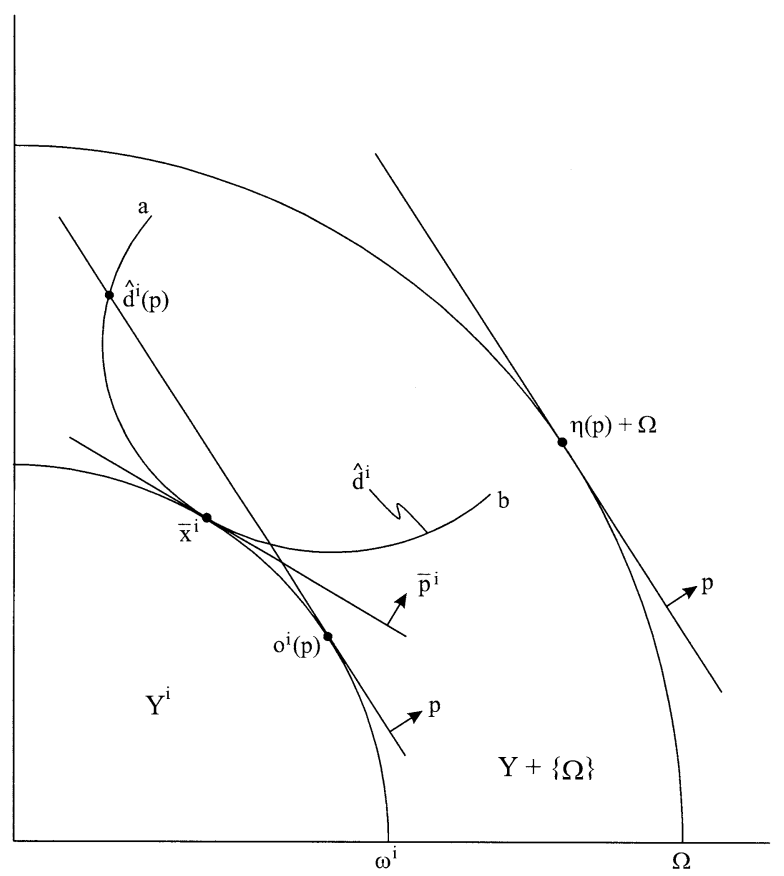

Fig. 1. Agent $i$ 's private production possibility set and modified offer curve

Then $\bar{x}^{i}=\widehat{d}^{i}\left(\bar{p}^{i}\right)$, and $\bar{p}^{i}$ separates $\widehat{d^{i}}$ from $Y^{i}$. Otherwise, for $p>\bar{p}^{i}, i$ 's demand $\widehat{d^{i}}(p)$ lies on the upper section of $\widehat{d}^{i}$ (between $\bar{x}^{i}$ and $a$ ), and for $p<\bar{p}^{i}, \widehat{d}^{i}(p)$ lies on the lower section (between $\bar{x}^{i}$ and $b$ ).

In the sequel, it will prove useful to consider agent $i$ 's excess demand function, or desired net trade, relative to its modified endowment. Thus, let $\hat{\xi}^{i}: \bar{P}^{i} \rightarrow \mathbb{R}^{m}$ be defined by $\widehat{\xi^{i}}(p) \equiv \widehat{d}^{i}(p)-o^{i}(p)$.

\section{The Walrasian demand manipulation game}

Under the Walrasian mechanism, each consumer announces its demand or, equivalently, excess demand. Since only agent $i$ knows $\widehat{\xi^{i}}$, it is possible that by announcing a function $\xi^{i}$ other than $\widehat{\xi}^{i}$ it might end up better-off. Hence, I formulate such announcements as a game wherein a strategy consists of a purported excess demand function, and Walrasian equilibria are computed on the basis of the purported excess demands.

I assume the announced excess demand functions must satisfy two (verifiable) restrictions. First, since the components of wealth are observable, net trades must be budget-balanced, $p \cdot \xi^{i}(p)=0$. And, second, the purported excess demands must satisfy a rationality condition. Specifically, they must satisfy a weak form 
of Samuelson's (1938) weak axiom of revealed preference as well as its transitive closure. Formally, for some $\widetilde{P}^{i} \subseteq \bar{P}$, I define the following: ${ }^{17}$

Definition 1. $\xi^{i}: \widetilde{P}^{i} \rightarrow \mathbb{R}^{m}$ satisfies the weak weak axiom of revealed preference (WWA) if for all $p, p^{\prime} \in \widetilde{P}^{i}, p \cdot \xi^{i}\left(p^{\prime}\right)<0$ implies $p^{\prime} \cdot \xi^{i}(p)>0 .{ }^{18}$

Definition 2. $\xi^{i}: \widetilde{P}^{i} \rightarrow \mathbb{R}^{m}$ satisfies the weak strong axiom of revealed preference (WSA) iffor every positive integer $r$ and for every sequence $\left\{p^{0}, p^{1}, \ldots, p^{r}\right\} \subset \widetilde{P}^{i}$, $p^{j+1} \cdot \xi^{i}\left(p^{j}\right)<0$, for all $j=0, \ldots, r-1$, implies $p^{0} \cdot \xi^{i}\left(p^{r}\right)>0 .{ }^{19,20}$

Definition 3. $\xi^{i}: \widetilde{P}^{i} \rightarrow \mathbb{R}^{m}$ is a strategic excess demand function for agent $i$ if it is continuous, budget-balanced, and satisfies WSA. ${ }^{21}$

Let $\Xi^{i}$ denote the set of strategic excess demand functions for agent $i$. As mentioned above, the Walrasian mechanism operates on the list $\xi=\left(\xi^{1}, \ldots, \xi^{n}\right) \in$ $\Xi^{1} \times \ldots \times \Xi^{n} \equiv \Xi$. Formally, a Walrasian equilibrium for $\xi$ is a price vector $p^{*} \in P$ and an allocation $\left(x^{*}, y^{*}\right) \in F$ such that $\sum_{i \in N} \xi^{i}\left(p^{*}\right)=0, y^{*}=\eta\left(p^{*}\right)$ and $x^{* i}=\xi^{i}\left(p^{*}\right)+o^{i}\left(p^{*}\right)$, for all $i \in N .^{22}$

Figure 2 depicts a Walrasian equilibrium for the case in which $n=m=2$. At prices $p^{*}$, the aggregate supply of resources is $y^{*}+\Omega=\eta\left(p^{*}\right)+\Omega$, and the profit to be distributed among the agents is $\pi\left(p^{*}\right)=p^{*} \cdot \eta\left(p^{*}\right)$. Agent 1 's consumption is measured from the origin and agent 2's is the residual from $\eta\left(p^{*}\right)+\Omega$. Since commodity 2 is taken to be numeraire, $\pi\left(p^{*}\right)$ is the vertical distance between $\Omega$ and the maximal isoprofit line through $\eta\left(p^{*}\right)+\Omega$.

As mentioned earlier, $B^{i}\left(p^{*}\right)$ is determined by the supporting hyperplane to $Y^{i}$ at $o^{i}\left(p^{*}\right)$. But $o^{1}\left(p^{*}\right)+o^{2}\left(p^{*}\right)=\eta\left(p^{*}\right)+\Omega$. Therefore, in Fig. 2, $p^{*}$ separates $Y^{1}$ from $Y^{2}$ (the latter measured from $\left.\eta\left(p^{*}\right)+\Omega\right)$ at $o\left(p^{*}\right) \equiv\left(o^{1}\left(p^{*}\right), o^{2}\left(p^{*}\right)\right)$, and both demands must lie on the mutual budget line. Finally, $\xi^{1}\left(p^{*}\right)+\xi^{2}\left(p^{*}\right)=0$,

17 Since, in principle, demand need not be defined over the entire price set, I allow for a "purported domain" other than $P . \widetilde{P}^{i}$ denotes the intersection of the purported domain with $\bar{P}$, just as $\bar{P}^{i}=P^{i} \cap \bar{P}$. Note, however, that for each of the demand functions constructed in the proof of Proposition 6, below, the domain is the entire set $\bar{P}$.

18 Applied to $d^{i}$, the weak axiom of revealed preference requires that if $p \cdot d^{i}\left(p^{\prime}\right) \leq p \cdot d^{i}(p)$ and $d^{i}\left(p^{\prime}\right) \neq d^{i}(p)$, then $p^{\prime} \cdot d^{i}\left(p^{\prime}\right)<p^{\prime} \cdot d^{i}(p)$. Whereas, the weak weak axiom, introduced by Kihlstrom et al. (1976), requires $p^{\prime} \cdot d^{i}\left(p^{\prime}\right)<p^{\prime} \cdot d^{i}(p)$ only in the event $p \cdot d^{i}\left(p^{\prime}\right)<p \cdot d^{i}(p)$. It is easy to verify that if $d^{i}$ is budget-balanced and satisfies the weak weak axiom, then $\xi^{i}$ satisfies WWA.

19 Generally, applied to $d^{i}$, the strong axiom of revealed preference, or the transitive closure of the weak axiom, is sufficient for rationalizability. Note, however, that for $m=2$, the weak axiom implies the strong axiom (see Rose 1958). Richter (1979) introduced the transitive closure of WWA, which he referred to as the weak weak strong axiom.

20 As in OS, WSA can be strengthened slightly to allow some, but not all, weak inequalities among the hypotheses.

21 Together with continuity and single-valuedness, WSA is sufficient to ensure that demand can be rationalized by a weak order (i.e., complete and transitive relation). To be precise, following Richter (1966), say $\xi^{i}(p)$ is indirectly revealed preferred to $\xi^{i}\left(p^{\prime}\right)$, written $\xi^{i}(p) R \xi^{i}\left(p^{\prime}\right)$, if there exists a sequence $\left\{p^{0}, p^{1}, \ldots, p^{r}\right\} \subset P$ such that $p^{\prime}=p^{0}, p=p^{r}$, and $p^{j+1} \cdot \xi^{i}\left(p^{j}\right)<0$ for all $j=$ $0, \ldots, r-1$. Then $R$ is irreflexive and transitive; hence, by Zorn's lemma, it has a complete and transitive extension, $\bar{R}$ (Richter 1966). $\bar{R}$ then rationalizes $\xi^{i}$ (see Mas-Colell 1982).

22 This requires that each of the functions $\xi^{i}$ and $\eta$ be defined at $p^{*}$, or that $p^{*} \in \bigcap_{i} \widetilde{P}^{i}$. 


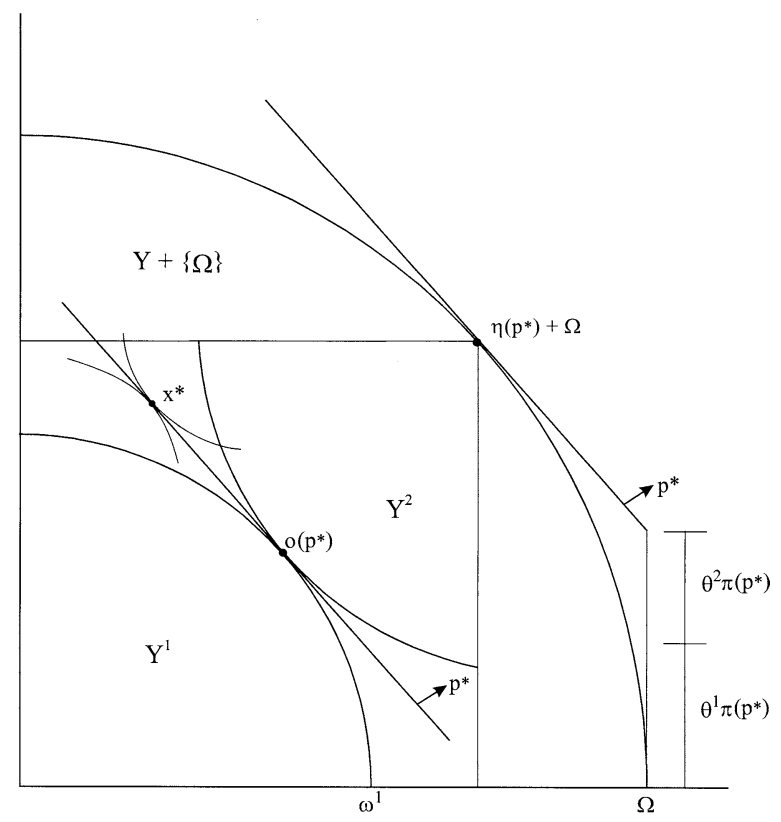

Fig. 2. A Walrasian equilibrium

where $\xi^{i}\left(p^{*}\right)=x^{* i}-o^{i}\left(p^{*}\right)$, i.e., the net trades are balanced. (The figure includes hypothetical indifference curves at $x^{*}$ that would rationalize the excess demands $\xi^{1}\left(p^{*}\right)$ and $\xi^{2}\left(p^{*}\right)$.)

There are two difficulties in defining the Walrasian demand manipulation game. First, taking $\Xi^{i}$ to be agent $i$ 's strategy set, the restrictions imposed by Definition 3 are not sufficient to ensure that there exists a Walrasian equilibrium for each $\xi \in \Xi$. For this reason, I will assume that in the event excess demands are inconsistent, no trade or production takes place. This will ensure that the outcome is well-defined. ${ }^{23}$

23 Given the properties of $\xi \in \Xi$ ascribed in Definition 3, it would be sufficient if aggregate excess demand $\zeta \equiv \sum_{i \in N} \xi^{i}$ were well-behaved as relative prices tend to zero, that is, if $\zeta$ were to satisfy a boundary condition such as that introduced by Neuefeind (1980) (see also Aliprantis and Brown 1983; Border 1985). If consumption were bounded below, then one might require that each $\xi^{i}$ satisfy such a boundary condition to ensure that $\zeta$ does as well. In particular, if an individual excess demand were to increase without bound, then the aggregate would do so too. However, with unbounded short sales, that is not so. It is possible that some consumers might wish to short-sell indefinitely large quantities of some commodities while others would wish to take infinitely long positions. Hence, the limit behavior of $\zeta$ might be indeterminate.

There are several recourses to rule out such behavior. First, one could simply impose restrictions directly on aggregate excess demand, as in the references cited above. A second recourse is to limit the degree of diversity among the agents. Various (related) formulations of such restrictions (on preferences) have been proposed including positive semi-independence (Hart 1974; Nielsen 1989), overlapping expectations (Hammond 1983), no unbounded arbitrage (Page 1987; Werner 1987; Chichilnisky 1995; Page and Wooders 1994), as well as various cone conditions discussed in Dana et al. (1999); Monteiro et al. (2000); or Aliprantis et al. (2000). A third strategy, appropriate for applying Negishi's (1960) method in the event preferences admit utility representations, is to impose restrictions directly on the set of feasible and individually rational utility allocations to ensure its compactness (cf. Cheng 1991; Dana et al. 1997; Page and Wooders 1996; Brown and Werner 1995). 
Next, even if Walrasian equilibria do exist, they need not be unique. Hence, it is necessary to specify an outcome correspondence rather than an outcome function. Formally, let $\Xi^{e} \subset \Xi$ denote the subset of consistent profiles, i.e., those for which there exists a Walrasian equilibrium. Then the Walrasian correspondence, $W^{e}$ : $\Xi^{e} \rightarrow P \times F$, associates with each $\xi \in \Xi^{e}$ its set of Walrasian equilibria. In light of the previous remark, I extend $W^{e}$ to the entire domain by assigning $P \times\{(\omega, 0)\}$ to each $\xi \in \Xi \backslash \Xi^{e}$. I denote the extended correspondence by $W$ and the projection of $W$ onto $F$ by $W_{F}$.

Definition 4. The Walrasian demand manipulation game is given by the triple $\Gamma=$ $\{N, \Xi, W\}$, where $N$ is the set of players, $\Xi$ is the strategy space, and $W: \Xi \rightarrow$ $P \times F$ is the outcome correspondence.

Given a profile $\xi \in \Xi$, since $W$ may be multivalued, it is not clear which (Walrasian) equilibrium would occur if agent $i$ were to change its demand announcement. Hence, it is not clear how to ascertain an optimal announcement. Following Otani and Sicilian, I adopt the notion of Nash equilibrium to the case of multivalued outcome functions by assuming that agents are "optimistic" in their assessment of alternative prospects, i.e., they will deviate if it is possible that they will end up better-off. ${ }^{24}$

Definition 5. A generalized Nash (GN) equilibrium is a list of strategies $\xi^{*} \in \Xi$ and an allocation $z^{*}=\left(x^{*}, y^{*}\right) \in F$ such that $z^{*} \in W_{F}\left(\xi^{*}\right)$ and for all $i \in N$, for all $\xi^{i} \in \Xi^{i}$ and for all $(x, y) \in W_{F}\left(\xi^{i}, \xi^{*-i}\right), x^{* i} \widehat{\succsim}_{i} x^{i}$.

The set of GN equilibria is denoted by $\mathcal{G N}$ and its projection onto $F$ by $\mathcal{G N}_{F}$. $z \in \mathcal{G} \mathcal{N}_{F}$ is called a $G N$ allocation.

\section{The set of GN allocations}

I begin this section by introducing some additional notation.

First, define the inverse supply correspondence $h: \partial Y \rightarrow \bar{P}$ by

$$
h(y)=\{p \in \bar{P} \mid y=\eta(p)\} .
$$

Also, agent $i$ 's (true) inverse demand correspondence $g: X^{i} \rightarrow P^{i}$ is given by

$$
g^{i}\left(x^{i}\right)=\left\{p \in P^{i} \mid x^{\prime i} \widehat{\succsim}_{i} x^{i} \text { and } x^{\prime i} \neq x^{i} \text { implies } p \cdot x^{i}>p \cdot x^{i}\right\} .
$$

Next, define the sets

$$
\begin{gathered}
U \equiv\left\{(x, y) \in F \mid \text { for all } i \in N, p^{i} \cdot x^{i}>p^{i} \cdot o^{i}\left(p^{i}\right) \text { for some } p^{i} \in g^{i}\left(x^{i}\right)\right\}, \\
T \equiv\left\{(x, y) \in \bar{F} \mid \text { for some } p \in h(y), p \cdot x^{i}=p \cdot o^{i}(p) \text { for all } i \in N\right\},
\end{gathered}
$$

In the present context, any restriction on individual demands based on aggregate considerations would be inappropriate. Consequently, rather than attempt to ensure that the manipulation mechanism is welldefined by requiring that a Walrasian equilibrium exists for each profile of excess demands, I modify the outcome rule to accomodate cases in which equilibria fail to exist (see also Desgranges et al. 2003).

24 This yields the smallest possible equilibrium set. For a discussion of alternative modifications for multivalued outcome functions see Thomson (1984). 


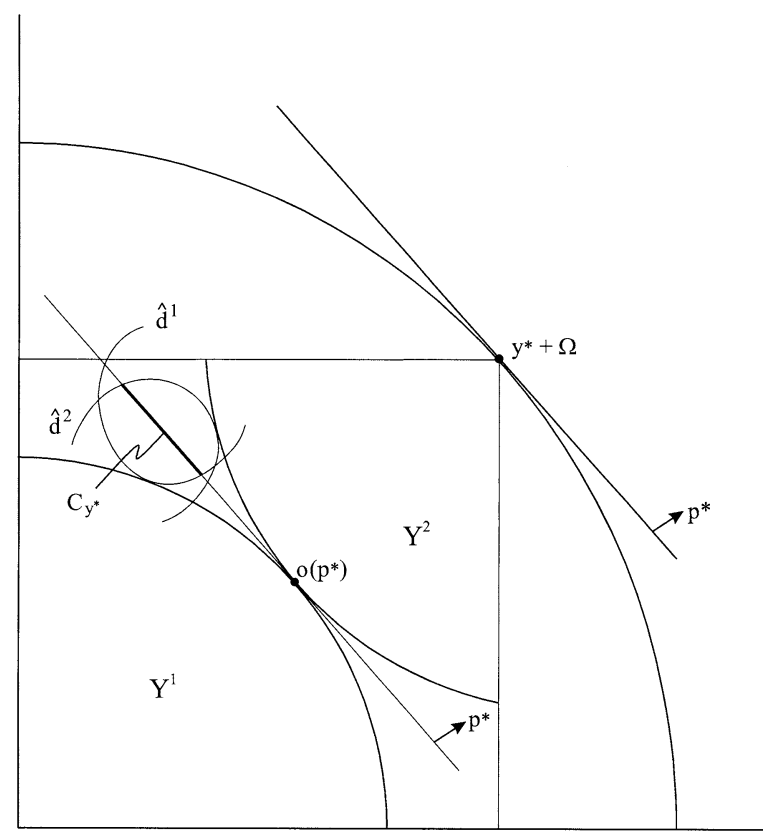

Fig. 3. The projection $C_{y^{*}}$

and

$$
C \equiv U \cap T
$$

Intuitively, $U$ is the set of feasible allocations at which each agent's consumption bundle lies above its true (modified) offer curve, and $T$ is the set of allocations that satisfy each agent's budget equation at the supply prices.

For $y \in \partial Y$, let $U_{y}, T_{y}$, and $C_{y}$ denote, respectively, the projections of $U, T$, and $C$ onto $X$ at $y$. Figure 3 depicts $C_{y^{*}}$.

Proposition 6. Under Assumptions 1 and 2, any allocation in $C$ can be supported as a $G N$ allocation, i.e., $C \subset \mathcal{G N}_{F}$.

Referring to Fig. 3, above, Proposition 6 establishes that the projection of $\mathcal{G N}_{F}$ at $y^{*}$ contains $C_{y^{*}}$.

In proving the proposition, I generalize the constructive argument of OS. First, let $z^{*}=\left(x^{*}, y^{*}\right) \in C$ be given, and take $p^{*} \in h\left(y^{*}\right)$ such that $p^{*} \cdot x^{* i}=p^{*} \cdot o^{i}\left(p^{*}\right)$, for all $i \in N$. (Such a $p^{*}$ exists by the definition of $T$.) Also, for all $i \in N$, let $p^{i} \in g^{i}\left(x^{* i}\right)$ be given such that $p^{i} \cdot x^{* i}>p^{i} \cdot o^{i}\left(p^{i}\right)$. (Here, too, the existence of $p^{i}$ follows from the definition of $U$.) I now construct excess demand functions $\xi^{*}$ such that $\left(x^{*}, y^{*}\right) \in W_{F}\left(\xi^{*}\right) .{ }^{25}$ I then show that these are well-behaved strategies and, finally, that they constitute a GN equilibrium.

25 Such a construction requires short-selling. That is, in the present context, continuity, budgetbalancedness and WSA are incompatible with the requirement that demand be nonnegative. 
Lemma 7. There exists a function $\psi: \bar{P} \rightarrow \mathbb{R}_{++}$such that $(i) \psi$ is continuous, (ii) $\psi\left(p^{*}\right)=1$, (iii) for all $i \in N$ and for all $p \in \bar{P}, p^{i} \cdot d^{* i}(p) \leq p^{i} \cdot x^{* i}$, where $d^{* i}$ is defined by

$$
d_{k}^{* i}(p)=o_{k}^{i}(p)+\psi(p)\left(x_{k}^{* i}-o_{k}^{i}\left(p^{*}\right)\right) \text { for } k=1, \ldots, m-1,
$$

and

$$
d_{m}^{* i}(p)=p \cdot o^{i}(p)-\sum_{k=1}^{m-1} p_{k} d_{k}^{* i}(p),
$$

and (iv) for every $p \in \bar{P}, p \cdot d^{* i}(p)=p \cdot o^{i}(p)$.

Proof. The proof proceeds as in OS, where the function $\psi: \bar{P} \rightarrow \mathbb{R}_{++}$is defined pointwise.

First, note that for $d^{* i}$ as defined in Eqs. (2)

$$
p^{i} \cdot d^{* i}(p)=p^{i} \cdot o^{i}(p)+\psi(p)\left(p^{i}-p\right) \cdot\left(x^{* i}-o^{i}\left(p^{*}\right)\right)
$$

and

$$
d_{m}^{* i}(p)=o_{m}^{i}(p)+\psi(p)\left(x_{m}^{* i}-o_{m}^{i}\left(p^{*}\right)\right)-\psi(p) p \cdot\left(x^{* i}-o^{i}\left(p^{*}\right)\right) .
$$

Therefore,

$$
\begin{gathered}
p^{i} \cdot d^{* i}(p) \leq p^{i} \cdot x^{* i} \text { if and only if } \\
p^{i} \cdot o^{i}(p)+\psi(p)\left(p^{i}-p\right) \cdot\left(x^{* i}-o^{i}\left(p^{*}\right)\right) \leq p^{i} \cdot x^{* i},
\end{gathered}
$$

or

$$
\begin{gathered}
p^{i} \cdot d^{* i}(p) \leq p^{i} \cdot x^{* i} \text { if and only if } \\
\psi(p)\left(p^{i}-p\right) \cdot\left(x^{* i}-o^{i}\left(p^{*}\right)\right) \leq p^{i} \cdot\left(x^{* i}-o^{i}(p)\right) .
\end{gathered}
$$

For each $p \in \bar{P}$, define $\Lambda^{i}(p) \equiv p^{i} \cdot\left(x^{* i}-o^{i}(p)\right)$ and $\Sigma^{i}(p) \equiv p \cdot\left(x^{* i}-o^{i}\left(p^{*}\right)\right)$. By hypothesis, $\Lambda^{i}\left(p^{i}\right)>0$. Then since $p^{i} \cdot o^{i}\left(p^{i}\right) \geq p^{i} \cdot o^{i}(p), \Lambda^{i}(p)>0$ as well. Also, since $\sum_{i=1}^{m} \Sigma^{i}(p) \equiv 0$, it follows that $\Sigma^{i}(p) \leq 0$ for some $i \in N$. Hence, $\left\{i \in N \mid \Lambda^{i}\left(p^{*}\right)-\Sigma^{i}(p)>0\right\} \neq \varnothing$ for all $p \in \bar{P}$.

Rewriting (3), $p^{i} \cdot d^{* i}(p) \leq p^{i} \cdot x^{* i}$ if and only if

$$
\psi(p)\left(\Lambda^{i}\left(p^{*}\right)-\Sigma^{i}(p)\right) \leq \Lambda^{i}(p) .
$$

Next, I ensure that (4) holds for all $p \in \bar{P}$.

Let $p \in \bar{P}$ be given. I have already established that if $\Sigma^{i}(p)=\Lambda^{i}\left(p^{*}\right)$, then $\Sigma^{i}(p)>\psi(p)\left(\Lambda^{i}\left(p^{*}\right)-\Sigma^{i}(p)\right)=0$. 
Alternatively, if $\Sigma^{i}(p)<\Lambda^{i}\left(p^{*}\right)$, then $\psi(p)\left(\Lambda^{i}\left(p^{*}\right)-\Sigma^{i}(p)\right) \leq \Lambda^{i}(p)$ if and only if $\psi(p) \leq \frac{\Lambda^{i}(p)}{\left(\Lambda^{i}\left(p^{*}\right)-\Sigma^{i}(p)\right)}$. In this case, I can ensure that $p^{i} \cdot d^{* i}(p) \leq p^{i} \cdot x^{* i}$ for every $i \in N$ by defining

$$
\psi(p) \equiv \min _{i \in N}\left\{\frac{\Lambda^{i}(p)}{\left(\Lambda^{i}\left(p^{*}\right)-\Sigma^{i}(p)\right)} \mid \Lambda^{i}\left(p^{*}\right)-\Sigma^{i}(p)>0\right\} .
$$

Finally, suppose $\Sigma^{i}(p)>\Lambda^{i}\left(p^{*}\right)$. Then providing $\psi(p) \geq 0, \psi(p)\left(\Lambda^{i}\left(p^{*}\right)-\right.$ $\left.\Sigma^{i}(p)\right) \leq 0<\Lambda^{i}(p)$. In particular, that is the case for $\psi(p)$ defined by (5).

By construction, therefore, $\psi(p)>0$ for all $p \in \bar{P}$. Also, since $o^{i}(p)$ is continuous by Assumption 2, it follows that $\Lambda^{i}$ and $\Sigma^{i}$, and thus $\psi$, are continuous as well. By hypothesis, $\Sigma^{i}\left(p^{*}\right)=0$ for all $i \in N$, and thus $\psi\left(p^{*}\right)=1$. Also by construction, $p^{i} \cdot d^{* i}(p) \leq p^{i} \cdot x^{* i}$, for all $p \in \bar{P}$. Finally, it is clear from (2) that $p \cdot d^{* i}(p)=p \cdot o^{i}(p)$ for all such $p$.

Lemma 8. Let $d^{* i}$ be defined by Eqs. (2). Then $\xi^{* i} \equiv d^{* i}-o^{i}$ satisfies WSA.

Proof. First, I establish that $d^{* i}$ in (2) satisfies WWA. Here, $\xi^{* i}=d^{* i}-o^{i}$ is given by

$$
\xi_{k}^{* i}(p)=\psi(p)\left(x_{k}^{* i}-o_{k}^{i}\left(p^{*}\right)\right) \text { for } k=1, \ldots, m-1,
$$

and

$$
\xi_{m}^{* i}(p)=-\psi(p) \sum_{k=1}^{m-1} p_{k}\left(x_{k}^{* i}-o_{k}^{i}\left(p^{*}\right)\right)
$$

For $p, p^{\prime} \in \bar{P}$,

$$
\begin{aligned}
p^{\prime} \cdot \xi^{* i}(p) & =\left(\sum_{k=1}^{m-1} p_{k}^{\prime} \psi(p)\left(x_{k}^{* i}-o_{k}^{i}\left(p^{*}\right)\right)\right)-\left(\psi(p) \sum_{k=1}^{m-1} p_{k}\left(x_{k}^{* i}-o_{k}^{i}\left(p^{*}\right)\right)\right) \\
& =\psi(p)\left(p^{\prime}-p\right) \cdot\left(x^{* i}-o^{i}\left(p^{*}\right)\right) .
\end{aligned}
$$

Therefore, since $\psi(p)>0$ by construction,

$$
p^{\prime} \cdot \xi^{* i}(p)<0 \text { if and only if }\left(p^{\prime}-p\right) \cdot\left(x^{* i}-o^{i}\left(p^{*}\right)\right)<0 .
$$

Similarly, since $\psi\left(p^{\prime}\right)>0$,

$$
p \cdot \xi^{* i}\left(p^{\prime}\right)>0 \text { if and only if }\left(p-p^{\prime}\right) \cdot\left(x^{* i}-o^{i}\left(p^{*}\right)\right)>0 .
$$

Comparing (7) and (8),

$$
p^{\prime} \cdot \xi^{* i}(p)<0 \text { if and only if } p \cdot \xi^{* i}\left(p^{\prime}\right)>0 .
$$

Thus, $\xi^{* i}$ satisfies WWA. To see that it satisfies WSA as well, consider a sequence $\left\{p^{0}, p^{1}, \ldots, p^{r}\right\} \subset \bar{P}$, for some $r$, and suppose $p^{j+1} \cdot \xi^{* i}\left(p^{j}\right)<0$, for all $j=0, \ldots, r-1$. Then by (7), $\left(p^{j+1}-p^{j}\right) \cdot\left(x^{* i}-o^{i}\left(p^{*}\right)\right)<0$, for all $j=0, \ldots, r-1$. Therefore, $\sum_{j=0}^{r-1}\left(p^{j+1}-p^{j}\right) \cdot\left(x^{* i}-o^{i}\left(p^{*}\right)\right)=\left(p^{r}-p^{0}\right) \cdot\left(x^{* i}-o^{i}\left(p^{*}\right)\right)<0$. Hence, again by (7), $p^{r} \cdot \xi^{* i}\left(p^{0}\right)<0$, and by (9), $p^{0} \cdot \xi^{* i}\left(p^{r}\right)>0$. 
Lemma 9. Let $\xi^{*}=\left(\xi^{* i}\right)_{i \in N}$, where $\xi^{* i}$ is defined by Eqs. (6). Then $\left(\xi^{*}, z^{*}\right) \in$ $\mathcal{G N}$.

Proof. By definition, $z^{*} \in F$ and $y^{*}=\eta\left(p^{*}\right)$. Also, by construction $d^{* i}\left(p^{*}\right)=x^{* i}$, or $\xi^{* i}\left(p^{*}\right)=x^{* i}-o^{i}\left(p^{*}\right)$, for all $i \in N$. Hence, $z^{*} \in W_{F}\left(\xi^{*}\right)$. I need only show that $x^{* i} \widehat{\succsim}_{i} x^{i}$ for all $x^{i}$ such that $z=(x, y) \in W_{F}\left(\xi^{i}, \xi^{*-i}\right) \cdot{ }^{26}$

First, assume $\left(\xi^{i}, \xi^{*-i}\right) \in \Xi^{e}$, that is, there exists a Walrasian equilibrium for the economy $\left(\xi^{i}, \xi^{*-i}\right)$. Then since the only possible Walrasian allocations are those consistent with $\xi^{*-i}$ at some prices, it is sufficient to show that for all $p \in \bar{P}$, $x^{* i} \widehat{\succsim}_{i}\left(o^{i}(p)-\sum_{j \neq i} \xi^{* j}(p)\right)$, or that

$$
p^{i} \cdot x^{* i} \geq p^{i} \cdot\left(o^{i}(p)-\sum_{j \neq i} \xi^{* j}(p)\right) \text {, for some } p^{i} \in g^{i}\left(x^{* i}\right) .
$$

Take $p^{i} \in g^{i}\left(x^{* i}\right)$ to be that given prior to Lemma 7. From (6) and the fact that $z^{*} \in W_{F}\left(\xi^{*}\right)$ it follows that $\sum_{j \in N} \xi_{k}^{* j}(p)=\psi(p) \sum_{j \in N}\left(x_{k}^{* j}-o_{k}^{j}\left(p^{*}\right)\right)=0$, for $k=1, \ldots, m-1$, and $\sum_{j \in N} \xi_{m}^{* j}(p)=-\psi(p) \sum_{k=1}^{m-1} \sum_{j \in N} p_{k}\left(x_{k}^{* j}-o_{k}^{j}\left(p^{*}\right)\right)=0$. Therefore, $-\sum_{j \neq i} \xi^{* j}(p)=\xi^{* i}(p)$, for all $p \in \bar{P}$. Hence, $-p^{i} \cdot \sum_{j \neq i} \xi^{* j}(p)=$ $p^{i} \cdot \xi^{* i}(p)$. By construction, $p^{i} \cdot \xi^{* i}(p) \leq p^{i} \cdot\left(x^{* i}-o^{i}(p)\right)$ for all $p \in \bar{P}$, which establishes (10).

Finally, if $\left(\xi^{i}, \xi^{*-i}\right) \in \Xi \backslash \Xi^{e}$, then agent $i$ is assigned its initial endowment $\omega^{i}$. Since $p^{i} \cdot x^{* i}>p^{i} \cdot o^{i}\left(p^{i}\right)=p^{i} \cdot \omega^{i}+\theta^{i} p^{i} \cdot \eta\left(p^{i}\right)$ and $p^{i} \cdot \eta\left(p^{i}\right) \geq 0, p^{i} \cdot x^{* i}>p^{i} \cdot \omega^{i}$. Hence $x^{* i} \widehat{\succsim}_{i} \omega^{i}$ as well.

Combining the results of Lemmas 7, 8 and 9 establishes Proposition 6.

Remark 1. In the singular case in which $Y=-\mathbb{R}^{m}$ (i.e., exchange) and is thus only weakly convex rather than strictly convex, the entire model - including the above construction - reduces precisely to that in OS with the exception of relaxing the nonnegativity constraint on demand. Hence, this generalizes the exchange result to allow short-selling.

Finally, I consider the (topological) size of the set of GN allocations. For this, I assume the technology and the true preferences are smooth and, furthermore, that $\bar{P}=P$, or that supply prices span the full range. ${ }^{27}$ To be precise, first define the normal manifold of $Y$ by $\Phi \equiv\left\{(p, y) \in \mathbb{R}^{m} \times \mathbb{R}^{m} \mid p \cdot y \geq p \cdot y^{\prime}\right.$ for all $\left.y^{\prime} \in Y\right\}$, and the projection of $\Phi$ onto its first component by $\Phi_{p}$. Then Assumptions 1 and 2 are modified as follows:

Assumption $1^{\prime}$. For all $i \in N, \widehat{\succsim}_{i}$ is complete, reflexive, transitive, continuous, strictly convex, strictly monotonic and $\mathcal{C}^{1} .^{28}$

26 In the event the excess demand announcements $\left(\xi^{i}, \xi^{*-i}\right)$ are inconsistent, this would include the allocation $(\omega, 0)$.

27 I require only that the true preferences be smooth. Therefore, the construction in Lemma 7 remains valid. Analogously, Otani and Sicilian (1982) assume that the actual preferences are strongly monotonic while the purported preferences need only be weakly monotonic.

28 See Mas-Colell (1985). 
Assumption $2^{\prime}$. $Y$ is closed, strictly convex and satisfies $-\mathbb{R}_{+}^{m} \subset Y$ and $Y \cap \mathbb{R}_{+}^{m}=$ $\{0\}$. Moreover, $\partial Y$ is a $\mathcal{C}^{1}$ manifold and $\Phi_{p}=\mathbb{R}_{++}^{m}$.

Proposition 10. Under Assumptions $1^{\prime}$ and $2^{\prime}, \mathcal{G N}_{F}$ contains an $n \times(m-1)$ dimensional subset.

(The proof of the proposition appears in the Appendix.)

Consequently, the set of GN allocations remains (topologically) large for all finite economies satisfying the above assumptions. ${ }^{29}$

\section{Conclusion}

In this paper, I have extended the analysis of Hurwicz (1979) and Otani and Sicilian $(1982,1990)$ of the Walrasian demand manipulation game to production economies with unbounded short-selling. The main results of the paper are Proposition 6, which partially characterizes the strategic outcomes resulting from misrepresentation of demand, and Proposition 10, which suggests that distortions resulting from strategic behavior may be significant even in large (finite) economies. Moreover, the generalization of the Nash equilibrium concept employed here affords the smallest evaluation of the set of strategic equilibrium allocations.

These arguments can be extended to economies with additional firms by vesting each consumer with a portion of every technology commensurate with its ownership share and then constructing its private production set as the sum of these component sets.

Also, as mentioned earlier, the results of our study might be applicable to a variety of areas, including manipulation in financial/asset or commodity markets, strategic behavior in international trade, and speculation in markets for foreign exchange. Indeed, interpreting short-selling in the manner suggested above, namely, as entailing the promise of future delivery ${ }^{30}$, the results might pertain in any intertemporal market setting.

\section{Appendix}

Proposition 10 is obtained immediately from Proposition 6 and the following lemma:

Lemma 11. Suppose $C \neq \emptyset$. Then there exists a set $\mathcal{V} \subset \partial Y$ that is open in $\partial Y$ such that for every $y \in \mathcal{V}$, there is a set $\mathcal{N}(y) \subset X$ that is open relative to $X$ such that $\mathcal{N}(y) \subset U_{y}$ and $\mathcal{N}(y) \cap T_{y}$ contains an $(n-1) \times(m-1)$-dimensional subset. Hence, $C$ contains an $n \times(m-1)$-dimensional subset.

\footnotetext{
29 The proposition does not preclude the possibility that the set of GN allocations might collapse in the limit to a set of smaller dimension. However, one can argue, as in OS, that that need not be so. Specifically, consider a replica economy with $r$ types of agents in which only coalitions consisting of one agent of each type have access to the aggregate technology and there are no other production possibilities. Then the construction in Lemma 7 remains valid, and the set of GN allocations will contain a subset of dimension $r(m-1)$ independent of the number of agents of each type.

30 See Footnote 10.
} 
Proof. The structure of the argument is as follows. First, I define a continuous mapping which associates with each $y$ in an open subset of $\partial Y$ an open subset of $U_{y}$ containing an $(n-1) \times(m-1)$-dimensional subset of $T_{y}$. Consequently, since the domain of the mapping is an $(m-1)$-dimensional submanifold of $\partial Y$, the graph is an $n \times(m-1)$-dimensional subset of $C$. In light of the feasibility constraints, this comprises a full-dimensional subset of the feasible set. ${ }^{31}$

To proceed, let $\rho(C)$ denote the projection of $C$ onto $Y$. That is, $\rho(C)=\{y \in$ $Y \mid(x, y) \in C$ for some $x \in X\}$. By definition $\rho(C) \subset \partial Y$, and by assumption $\rho(C) \neq \emptyset$. For now, take $\bar{y} \in \rho(C)$ as given and let $\bar{x} \in C_{\bar{y}}$. Since $h$ is single-valued, $h(\bar{y}) \cdot\left(\bar{x}^{i}-o^{i}(h(\bar{y}))\right)=0$, for all $i \in N .^{32}$

Define $G^{i}: X^{i} \rightarrow \mathbb{R}$ by $G^{i}\left(x^{i}\right)=g^{i}\left(x^{i}\right) \cdot\left(x^{i}-o^{i}\left(g^{i}\left(x^{i}\right)\right)\right)$. To see that $G^{i}$ is continuous, notice that it can be written as $\lambda^{i} \circ \gamma^{i}$, where $\gamma^{i}: X^{i} \rightarrow P \times X^{i}$ is given by $\gamma^{i}\left(x^{i}\right)=\left(g^{i}\left(x^{i}\right), x^{i}\right)$ and $\lambda^{i}: P \times X^{i} \rightarrow \mathbb{R}$ is given by $\lambda^{i}\left(p, x^{i}\right)=$ $p \cdot\left(x^{i}-o^{i}(p)\right)$. Under Assumption $2^{\prime}, o^{i}(p)$ is defined for all $p \in P$, hence $\lambda^{i}$ is well-defined. Under $1^{\prime}$ and $2^{\prime}$, both $\lambda^{i}$ and $\gamma^{i}$ are continuous.

Since $G^{i}$ is continuous, $\mathcal{N}^{i} \equiv\left\{x^{i} \in X^{i} \mid G^{i}\left(x^{i}\right)>0\right\}$ is open in $X^{i}$. Let $\mathcal{N}=\mathcal{N}^{1} \times \ldots \times \mathcal{N}^{n}$. Then since $\bar{x} \in \mathcal{N}$, there is an open neighborhood of $\bar{x}$, denoted $\mathcal{N}(\bar{y}) \subset \mathcal{N}$, such that $\mathcal{N}(\bar{y}) \subset U_{\bar{y}}$.

Next, consider the linear mapping from $\mathbb{R}^{m(n-1)}$ to $\mathbb{R}^{n-1}$ defined by $A(\bar{y}) v$, where $A(\bar{y})=h(\bar{y})^{t}$ and $v=\left(z^{1} \cdots z^{n-1}\right) \in \mathbb{R}^{m(n-1)}$, treating $z^{i}$ and $h(\bar{y})$ as $(m \times 1)$ column vectors.

The kernel of $A(\bar{y})$ is given by $Z(\bar{y}) \equiv\left\{v \in \mathbb{R}^{m(n-1)} \mid A(\bar{y}) v=0_{n-1}\right\}$, where $0_{n-1}$ denotes the $1 \times(n-1)$ zero matrix. Then $\bar{v}=\left(\bar{z}^{1} \ldots \bar{z}^{n-1}\right) \in Z(\bar{y})$, where $\bar{z}^{i}=\left(\bar{x}^{i}-o^{i}(h(\bar{y}))\right)$. Hence, $\operatorname{dim} Z(\bar{y})=m(n-1)-(n-1)=(n-1)(m-1)$. And if $v \in Z(\bar{y})$, then taking $z^{n}=-\sum_{j \neq i} z^{j},(o(h(\bar{y}))+z) \in T_{\bar{y}}$. Thus, $\mathcal{N}(\bar{y}) \cap$ $T_{\bar{y}}$ contains an $(n-1)(m-1)$-dimensional subset.

Finally, since $\bar{y} \in \rho(C)$ was arbitrary, I need only show that $\rho(C)$ contains an open subset.

Let $H: \partial Y \rightarrow X$ be defined by $H(y)=T_{y}$. That is, $H$ associates with each $y \in \partial Y$ the feasible consumption allocations that are budget-balanced at the supply prices $h(y)$. Clearly, under Assumption $2^{\prime}, H$ is continuous. Next, let $G=\left(G^{1}, \ldots, G^{n}\right)$, where $G^{i}$ is as defined above. Thus $G: X \rightarrow \mathbb{R}^{n}$. I have shown that $G$ is continuous. Therefore, $G \circ H$ is an upper hemi-continuous correspondence. By Hildenbrand (1974, Proposition 1, p. 22), $\mathcal{V} \equiv\{y \in \partial Y \mid(G \circ H)(y) \gg 0\}$ is open in $\partial Y$. Moreover, by construction, $\mathcal{V} \subset \rho(C)$.

31 Generally, in an exchange environment, an $n \times m$-dimensional feasible set can be described parametrically in $(n-1) \times m$ dimensions by taking the consumption of one agent to be the residual of that of the others. (Hence, the 2-dimensional Edgeworth box depicts a 4-dimensional feasible set.) Here, for given $y \in Y$, the projection of the feasible set $F$ at $y$ might thus be described parametrically as an $(n-1) \times m$-dimensional set. Letting $y$ vary over an open subset of $\partial Y$ results in a set of dimension $n \times m$. Finally, restricting the set by requiring budget-balancedness (Walras's law), i.e., restricting attention to $T_{y}$, the dimensionality would be reduced to $n \times(m-1)$.

32 Here, I use $h(y)$ to denote the unique supporting price vector. Similarly, $g^{i}\left(x^{i}\right)$ denotes the unique normal to the $\widehat{\succsim}_{i}$-indifference surface at $x^{i}$. 
Hence, there exists an open subset $\mathcal{V}$ of $\rho(C) \subset \partial Y$. And since $\partial Y$ is homeomorphic to an $(m-1)$-manifold, $\operatorname{dim} \mathcal{V}=m-1$. Therefore, $C$ contains an $(m-1)(n-1)+(m-1)=n(m-1)$ dimensional subset.

\section{References}

Aliprantis, C.D., Brown, D.J. (1983) Equilibria in markets with a Reisz space of commodities. Journal of Mathematical Economics 11: 189-207

Aliprantis, C.D., Tourky, R., Yannelis, N. (2000) Cone conditions in general equilibrium theory. Journal of Economic Theory 92: 96-121

Border, K. (1985) Fixed Point Theorems with Applications to Economics and Game Theory. Cambridge University Press, Cambridge

Brown, D.J., Werner, J. (1995) Arbitrage and existence of equilibrium in infinite asset markets. Review of Economic Studies 62: 101-114

Cheng, H. (1991) Asset market equilibrium in infinite dimensional complete markets. Journal of Mathematical Economics 20: 137-152

Chichilnisky, G. (1995) Limited arbitrage in necessary and sufficient for the existence of a competitive equilibrium with and without short sales. Economic Theory 5: 79-108

Chichilnisky, G., Heal, G.M. (1993) Competitive equilibrium in Sobolev spaces without bounds on short sales. Journal of Economic Theory 59: 364-384

Dana, R.-A., Le Van, C., Magnien, F. (1997) General equilibrium in asset markets with or without short-selling. Journal of Mathematical Analysis and Applications 206: 567-588

Dana, R.-A., Le Van, C., Magnien, F. (1999) On different notions of arbitrage and existence of equilibrium. Journal of Economic Theory 87: 169-193

Desgranges, G., Geoffard, P.-Y.; Guesnerie, R. (2003) Do prices transmit rationally expected information? Journal of the European Economic Association 1: 124-153

Gabszewicz, J., Vial, J.-P. (1972) Oligopoly 'a la Cournot' in a general equilibrium analysis. Journal of Economic Theory 4: $381-400$

Groves, T., Ledyard, J. (1987) Incentive compatibility since 1972. In: Groves, T., Radner, R., Reiter, S. (eds.) Information, Incentives, and Economic Mechanisms: Essays in Honor of Leonid Hurwicz. University of Minnesota Press, Minneapolis

Hammond, P.J. (1983) Overlapping expectations and Hart's conditions for equilibrium in a securities model. Journal of Economic Theory 31: 170-175

Hart, O.D. (1974) On the existence of equilibrium in a securities model. Journal of Economic Theory 9: 293-311

Hildenbrand, W. (1974) Core and Equilibria of a Large Economy. Princeton University Press, Princeton

Hong, L. (1995) Nash implementation in production economies. Economic Theory 5: 401-417

Hurwicz, L. (1972) On informationally decentralized systems. In: McGuire, C.B., Radner, R. (eds.) Decision and Organization. North-Holland, Amsterdam

Hurwicz, L. (1979) On the interaction between information and incentives. In: Krippendorff, K. (ed.) Communication and Control in Society. Gordon \& Breach, New York

Jackson, M. (1992) Incentive compatibility and competitive allocations. Economics Letters 40: 299-302

Kihlstrom, R., Mas-Colell, A., Sonnenschein, H. (1976) The demand theory of the weak axiom of revealed preference. Econometrica 44: 971-978

Kranich, L. (1992) Manipulation of the Walrasian mechanism in simple production economies. Mimeo, Penn State University, USA

Mas-Colell, A. (1982) Revealed preference after Samuelson. In: Feiwel, G. (ed.) Samuelson and Neoclassical Economics. Kluwer-Nijhoff, Boston

Mas-Colell, A. (1985) The Theory of General Economic Equilibrium: A Differentiable Approach. Cambridge University Press, Cambridge

Maskin, E. (1985) The theory of implementation in Nash equilibrium: A survey. In: Hurwicz, L., Schmeidler, D., Sonnenschein, H. (eds.) Social Goals and Social Organization: Essays in Memory of Elisha Pazner. Cambridge University Press, Cambridge 
Monteiro, P.K., Page, F.H. Jr., Wooders, M. (2000) Increasing cones, recession cones and global cones. Optimization 47: 211-234

Negishi, T. (1960) Welfare economics and existence of equilibrium for a competitive economy. Metroeconomica 12: 92-97

Neuefeind, W. (1980) Notes on existence of equilibrium proofs and the boundary behavior of supply. Econometrica 48: 1831-1837

Nielsen, L.T. (1989) Asset market equilibrium with short-selling. Review of Economic Studies 56: 467-474

Otani, Y. (1996) Consumption allocations and real indeterminacyof manipulative equilibrium in a strategic Walrasian market game. The Japanese Economic Review 47: 210-225

Otani, Y. (2001) The structure of equilibrium consumption allocations of a Lindahl strategic game. Discussion Paper No. 10, Faculty of Economics, Kyushu Sangyo University, Japan (http://www.ip.kyusan-u.ac.jp/J/keizai/prod02.htm)

Otani, Y., Sicilian, J. (1982) Equilibrium allocations of Walrasian preference games. Journal of Economic Theory 27: 47-68

Otani, Y., Sicilian, J. (1990) Limit properties of equilibrium allocations of Walrasian strategic games. Journal of Economic Theory 51: 295-312

Page, F.H. Jr. (1987) On equilibrium in Hart's securities exchange model. Journal of Economic Theory 41: 392-404

Page, F.H. Jr., Wooders, M.H. (1994) Arbitrage in markets with unbounded short sales: necessary and sufficient conditions for nonemptiness of the core and existence of equilibrium. Mimeo, University of Toronto, Canada

Page, F.H. Jr., Wooders, M.H. (1996) A necessary and sufficient condition for compactness of individually rational and feasible outcomes and existence of an equilibrium. Economics Letters 52: $153-162$

Postlewaite, A. (1985) Implementation via Nash equilibria in economic environments. In: Hurwicz, L., Schmeidler, D., Sonnenschein, H. (eds.) Social Goals and Social Organization: Essays in Memory of Elisha Pazner. Cambridge University Press, Cambridge

Rader, T. (1972) Theory of Microeconomics. Academic Press, New York

Richter, M. (1966) Revealed preference theory. Econometrica 34: 635-645

Richter, M. (1979) Duality and rationality. Journal of Economic Theory 20: 131-181

Roberts, D.J., Postlewaite, A. (1976) The incentives for price-taking behavior in large exchange economies. Econometrica 44: 115-127

Rose, H. (1958) Consistency of preference: the two-commodity case. Review of Economic Studies 25: $124-125$

Samuelson, P. (1938) A note on the pure theory of consumer behavior. Economica 5: 61-71

Samuelson, P. (1954) The pure theory of public expenditure. Review of Economics and Statistics 36: 387-389

Sobel, J. (1981) Distortion of utilities and the bargaining problem. Econometrica 49: 597-619

Sobel, J. (1998) Manipulation of preferences and relative utilitarianism. Mimeo, University of California, San Diego, USA

Thomson, W. (1979) The equilibrium allocations of Walras and Lindahl manipulation games. Mimeo, University of Minnesota, USA

Thomson, W. (1984) The manipulability of resource allocation mechanisms. Review of Economic Studies 51: 447-460

Werner, J. (1987) Arbitrage and the existence of competitive equilibrium. Econometrica 55: 1403-1418

Zhou, L. (1991) Inefficiency of strategy-proof allocation mechanisms in pure exchange economies. Social Choice and Welfare 8: 247-254 